Revue d'histoire de l'Amérique française

REVUE D.HISTOIRE DE L'AMÉRIQUE FRANÇAISE

\title{
Élites locales, institutions et fonctions publiques dans la paroisse de Saint-Roch-de-l'Achigan, de 1810 à 1840
}

\section{Jean-René Thuot}

Volume 57, numéro 2, automne 2003

URI : https://id.erudit.org/iderudit/009142ar

DOI : https://doi.org/10.7202/009142ar

Aller au sommaire du numéro

Éditeur(s)

Institut d'histoire de l'Amérique française

ISSN

0035-2357 (imprimé)

1492-1383 (numérique)

Découvrir la revue

Citer cet article

Thuot, J.-R. (2003). Élites locales, institutions et fonctions publiques dans la paroisse de Saint-Roch-de-l'Achigan, de 1810 à 1840 . Revue d'histoire de l'Amérique française, 57(2), 173-208. https://doi.org/10.7202/009142ar
Résumé de l'article

La présente recherche se propose de jeter un regard sur l'ensemble des institutions et fonctions publiques de la paroisse de Saint-Roch-de-l'Achigan entre 1810 et 1840 . Après avoir passé en revue les différentes charges publiques qui se retrouvaient dans cette paroisse rurale au début du XIX ${ }^{\mathrm{e}}$ siècle, le texte considère les personnes qui les ont occupées. Cette démarche permet de jumeler un profil socio-économique à chaque fonction publique et, à travers cette exploration de l'origine des acteurs, de faire émerger une définition d'une élite institutionnelle. 


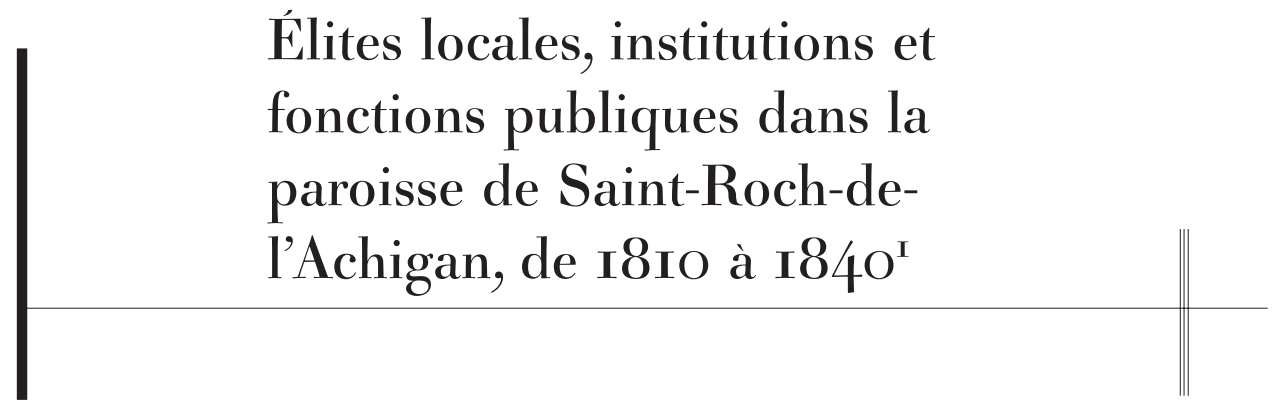

JEAN-RENÉ THUOT

Département d'histoire

Université de Montréal

RÉSUMÉ - La présente recherche se propose de jeter un regard sur l'ensemble des institutions et fonctions publiques de la paroisse de Saint-Roch-de-l'Achigan entre 1810 et 1840. Après avoir passé en revue les différentes charges publiques qui se retrouvaient dans cette paroisse rurale au début du XIXe siècle, le texte considère les personnes qui les ont occupées. Cette démarche permet de jumeler un profil socio-économique à chaque fonction publique et, à travers cette exploration de l'origine des acteurs, de faire émerger une définition d'une élite institutionnelle.

ABSTRACT - This research project suggests a general overview of public functions and institutions of the Saint-Roch-de-l'Achigan parish between the years 1810-1840. The text first offers an examination of the public duties and charges as they existed in the parish during the above-mentioned time period. It then integrates the people who occupied these positions in the analysis. This approach allows to associate given socioeconomic profiles to the exercise of specific duties and charges, and thus suggests a definition of institutional elites that finds its basis in the examination of individual actors' origins.

1. Le présent article puise au contenu d'un mémoire rédigé sous la direction de Christian Dessureault. Voir Jean-René Thuot, Élites locales, institutions et fonctions publiques à Saint-Roch-del'Achigan, de 1810 à 1840, mémoire de maîtrise (histoire), Université de Montréal, 2003, 198 p. L'auteur tient à remercier conjointement Denyse Baillargeon, son directeur Christian Dessureault et les évaluateurs anonymes pour leurs précieux conseils et suggestions, qui ont permis de bonifier le présent article. 
T es ÉTUdes DES DERNIÈRES DÉCENNIES sur le Québec préindustriel ont Ldémontré la complexité du tissu social des communautés rurales. À la lumière de ces travaux, il apparaît opportun d'examiner de manière plus générale l'interaction entre les structures sociales et les structures de pouvoir dans ces communautés. Pour mieux saisir cette interaction, nous avons choisi de nous pencher sur la communauté de Saint-Roch-del'Achigan pour la période 1810-1840, pour laquelle nous avons procédé à une étude par étape : observation du recrutement propre à chaque institution ou charge publique, classement des diverses fonctions publiques locales et jumelage avec les profils socio-économiques des acteurs publics. Cette approche du «local», qui permet de faire émerger une définition des élites institutionnelles, s'inspire des problématiques et des méthodes développées depuis une vingtaine d'années aux États-Unis, en France, en Grande-Bretagne et au Canada, et qui sont venues enrichir l'étude des petites communautés ${ }^{2}$. Dans son article publié en 1986, Darrett B. Rutman ${ }^{3}$ insiste sur l'importance des structures dans l'étude des communautés locales. Selon lui, s'il y a forcément des différences de "vécu» d'une communauté à l'autre, chacune d'entre elles peut se comprendre en suivant le même schéma d'ensemble. Comme ce dernier, nous voulons, à partir d'un cas exemplaire, cerner une dynamique d'évolution transcendant notre terrain d'enquête. Plus précisément, à partir d'une étude locale, nous visons à mettre en lumière une partie des mécanismes d'articulation du social à l'institutionnel dans le Québec rural préindustriel.

D’entrée de jeu, il convient de souligner la maigre production traitant de l'ensemble des fonctions publiques d'une localité, une carence observable autant du côté de l'historiographie américaine, française, anglaise que canadienne. Dans la majorité des études, en effet, les historiens ont examiné la composition sociale de corpus d'individus se rattachant à une ou deux fonctions publiques locales, quelquefois davantage, mais rarement de façon exhaustive.

2. À ce titre, notre démarche tient entre autres compte de l'observation de Béatrice Craig, qui soulignait que notre historiographie «s'est beaucoup nourrie d'emprunts venant de France», ne s'ouvrant «aux influences nord-américaines » que lentement, ce qui invite à un rééquilibrage. Béatrice Craig, "Pour une approche comparative de l'étude des sociétés rurales nord-américaines ", Histoire sociale/Social History, 23,46 (1990): 254.

3. Darrett B. Rutman, "Assessing the Little Communities", The William and Mary Quarterly, 63,2 (avril 1986): 163-178. 
D’une part, dans les cas où les études embrassent plusieurs institutions ${ }^{4}$, le portrait social est souvent limité en raison du peu d'attention portée aux réseaux familiaux et sociaux. La force de ces ouvrages repose sur l'importance accordée à la composition socio-économique de chacun des groupes sociaux présents aux postes de pouvoir. Ils visent à établir des modèles, en scrutant les niveaux de fortune, la superficie des terres, le nombre d'années en poste et le cumul des fonctions. Ces études établissent une hiérarchie entre les fonctions électives et proposent des modèles d'évolution - ou de reproduction.

D'autre part, certaines études locales se concentrent sur de petits échantillons ou sur certaines institutions particulières — dans le moyen et le long terme —, de manière à mieux faire ressortir les interactions entre les dimensions sociale et institutionnelle $e^{5}$. Toutefois, la plupart de ces travaux ne détaille pas suffisamment l'éventail des charges publiques et évacue la dimension des rapports que ces charges entretiennent entre elles. Les auteurs consacrent une bonne partie de l'analyse à vérifier les trajectoires de groupes familiaux ou d'individus rattachés à certaines charges publiques trop souvent les mieux documentées, ou les plus en vue. La précision des profils sociaux des acteurs représente certes un avantage de cette voie, mais la vue d'ensemble y perd au change.

En bout de ligne, le constat est le même: plusieurs études socioinstitutionnelles concentrent leurs énergies sur quelques lieux de pou-

4. Voir entre autres David Eastwood, Governing Rural England: Tradition and Transformation in Local Government, 1780-1840 (New York, Oxford University Press, 1994), 311 p. ; Christian Kermoal, Les notables du Trégor: éveil à la culture politique et évolution dans les paroisses rurales (17701850), (Rennes, Presses universitaires de Rennes, 2002) 488 p. ; Mark Goldie, «The Unacknowledged Republic: Officeholding in Early Modern England", dans Tim Harris, dir., The Politics of the Excluded, c.1500-1850 (London, Basingstoke: Palgrave, 2001), 153-194; Trevor Burnard, Creole Gentlemen. The Maryland Elite, 1691-1776 (New York, Routhledge, 2002), 278 p.

5. Voir entre autres Guy Saupin, «Les oligarchies municipales en France sous l'Ancien Régime: réflexion méthodologique sur l'analyse historique de leur reproduction à partir de l'exemple de Nantes", dans Claude Petitfrère, dir., Construction, reproduction et représentation des patriciats urbains de l'Antiquité au xxe siècle (Actes du colloque de Tours en 1998, CEHVI, Centre d'histoire de la ville moderne et contemporaine, 1999), 95-112; Eric Carlson, "The Origins, Function, and Status of the Office of Churchwarden, with Particular Reference to the Diocese of Ely», dans Margaret Spufford, dir., The World of Rural Dissenters, 1520-1725 (Cambridge, Cambridge University Press, 1995), 164-207 ; J. K. Johnson, Becoming Prominent-Regional Leadership in Upper Canada, 1791-1841 (Montréal, McGill-Queen's University Press, 1989), 277 p.; Robert Descimon, «Les capitaines de la milice bourgeoise à Paris (1589-1651): pour une prosopographie de l'espace social parisien ", dans Jean-Pierre Genet, dir., L'État moderne et les élites, XIIIXVIII siècles - Apports et limites de la méthode prosopographique - Actes du colloque international CNRSParis I, 16-19 octobre 1991 (Paris, Publications de la Sorbonne, 1996), 189-211. 
voir plutôt que sur l'enchevêtrement de plusieurs institutions considérées comme un tout. Le portrait social des acteurs varie d'un ouvrage à l'autre, en fonction du nombre d'institutions à l'étude.

Au Québec, l'étude du pouvoir institutionnel local présente jusqu'à maintenant plusieurs similitudes avec ce qui s'est fait ailleurs, avec les mêmes forces et les mêmes faiblesses. C'est ainsi qu'un certain nombre de travaux ont exploré à fond une seule institution locale ${ }^{6}$ : les appareils municipal et judiciaire, la milice ou la fabrique.

Donald Fyson, par exemple, a détaillé les fonctions et le profil social des juges de paix de la région de Montréal, entre 1764 et 1830. Il a aussi tracé un profil des jurys appelés à siéger aux tribunaux du district de Montréal entre 1764 et $1832^{7}$. De son côté, J. I. Little examine la mise en place de l'appareil municipal dans les Cantons de l'Est dans les années suivant le rapport Durham ${ }^{8}$. Christian Dessureault et Roch Legault, qui s'intéressent à la milice sédentaire de la région de Saint-Hyacinthe entre 1808 et 1830, offrent probablement l'étude sociale la plus approfondie d'une seule institution. Allan Greer aborde également la structure milicienne à travers une analyse des liens entre «l'habitant et l'État», mais il s'attarde davantage aux aspects structurel et symbolique de l'institution, plutôt qu'à définir les profils sociaux des acteurs? ${ }^{9}$ Pour ce qui est des études portant sur la fabrique, les articles d'Allan Greer et d'André Boucher s'attardent à définir les règles et mécanismes qui régissent cette institution, tandis que les travaux de Bruno Lamour, de Claudia Lohse-

6. Certains travaux essaient de brosser un tableau d'ensemble des institutions locales au BasCanada avant 1840, mais celui-ci demeure encore partiel et plutôt descriptif. Michel Monette, "Groupes dominants et structure locale de pouvoir à Deschambault et Saint-Casimir, comté de Portneuf (1829-1870)», Cahiers de géographie du Québec, 28,73-74 (avril-septembre 1984): 3-88; D. Fyson, «La paroisse et l'administration étatique sous le régime britannique (1764-1840)», dans Serge Courville, dir., Atlas historique du Québec - la paroisse (Québec, Les Presses de l’Université Laval, 2001), 25-37.

7. Donald Fyson, Criminal Justice, Civil Society and the Local State: The Justices of the Peace in the District of Montreal, 1764-1830, thèse de Ph.D. (Histoire), Université de Montréal, 1995, 465 p. ; voir également Donald Fyson, "Jurys, participation civique et représentation au Québec et au Bas-Canada: les grands jurys du district de Montréal (1764-1832)", Revue d'histoire de l'Amérique française, 55,1 (été 2001): 85-120.

8. J. I. Little, State and Society in Transition: The Politics of Institutional Reform in the Eastern Townships, 1838-1852 (Montréal, McGill-Queen’s University Press, 1997), 350 p.

9. Christian Dessureault et Roch Legault, «Évolution organisationnelle et sociale de la milice sédentaire canadienne: le cas du bataillon de Saint-Hyacinthe, 1808-1830", Journal of Canadian Historical Association/Revue de la société historique du Canada, 7 (1997): 87-112; voir également Allan Greer, "L'habitant et l'État», dans Habitants et patriotes. La Rébellion de 1837 dans les campagnes du Bas-Canada (Montréal, Boréal), 87-114 et 330-332. 
Busch ou du duo Christian Dessureault et Christine Hudon concourent à donner un visage plus social à la fabrique, par le biais de l'étude des marguilliers et des conflits pour le contrôle de l'institution ${ }^{10}$.

$\mathrm{Au}$ total, si elles nous renseignent sur la composition sociale de chacune des institutions considérées, certaines études québécoises, tout comme les travaux américains, français, anglais et canadiens, nous poussent à dresser un certain nombre de constats qui peuvent se traduire en autant de pistes de recherche. Il s'agit dans un premier temps de reconnaître la nécessité de hiérarchiser les fonctions publiques entre elles; dans un second temps, de ne jamais perdre de vue le portrait d'ensemble de ces fonctions et des acteurs qui les animent, saisies dans leurs interrelations; dans un troisième temps, de tenir compte de la notoriété locale comme facteur de différenciation sociale; dans un quatrième temps, de porter attention à la présence de clans ou de groupes d'intérêt au sein de la structure institutionnelle; et enfin, dans un cinquième temps, d'être sensible aux mécanismes d'articulation du social à l'institutionnnel. Nous croyons que la mise en lumière de ces mécanismes permettrait de faire ressortir la complexité sociale d'une localité, reflet d'une structure étatique informelle tout aussi complexe.

Ces pistes nous ont conduit à suivre l'évolution de la totalité des individus - sur un mode prosopographique - ayant exercé une fonction publique à l'intérieur des limites de Saint-Roch-de-l'Achigan, dans un espace-temps qui s'apparente au moyen terme. En bout de ligne, cette démarche devrait permettre de repérer une certaine "élite institutionnelle », c'est-à-dire un groupe à qui la communauté confère la légitimité nécessaire pour défendre ses intérêts. Pour parvenir à définir les contours d'une certaine condition élitaire liée à l'exercice du pouvoir local, nous avons cherché à ratisser un espace communautaire qui soit le plus

10. André Boucher, «La fabrique et les marguilliers», et "Le rôle joué par les marguilliers», dans Pierre Hurtubise, Le lä̈c dans l'Église canadienne-française de 1830 à nos jours (Montréal, Fides, coll. «Histoire religieuse du Canada », 1972), 147-175; Christian Dessureault et Christine Hudon, "Conflits sociaux et élites au Bas-Canada : le clergé, les notables, la paysannerie et le contrôle de la fabrique ", The Canadian Historical Review, 80,3 (septembre 1999) : 413-439; Allan Greer, "L'habitant, la paroisse rurale et la politique locale au $18^{\mathrm{e}}$ siècle", Société canadienne de l'histoire de l'Église catholique, 47 (1980): 19-33; Bruno Lamour, Une forme de notabilité villageoise: les marguilliers de Charlesbourg, 1675-1850, mémoire de maîtrise, Université de Haute-Bretagne Rennes II, 1989, 172 p.; Claudia Lohse-Busch., Étude sociale de l'institution de la fabrique: le cas de deux paroisses du Bas-Richelieu au XVIII siècle, Saint-Antoine et Saint-Denis, mémoire de maîtrise, Université des Sciences humaines de Strasbourg (recherche et rédaction effectuées à l'Université de Montréal), 1998, 140 p. 
large possible - précisément pour élargir l'éventail des "possibles». Aussi, la définition de "fonction publique» retenue ici renvoie à quelque chose de plus large que la simple expression étatique du pouvoir au niveau local. Pour les fins de l'analyse, nous considérons donc autant les charges étatiques, para-étatiques et paroissiales; en fait, la définition de "charge publique» proposée ici embrasse toute fonction institutionnelle liée à la gestion de l'espace communautaire local. Ces fonctions sont "publiques" au sens où elles sont accessibles à tous théoriquement, sans formation préalable. On parle ici de postes de pouvoir pour lesquels il y a concurrence ou sélection entre agents sociaux de différentes formations qui, en théorie, ont tous un accès égal à ces postes. Lorsqu'on désigne les fonctions institutionnelles comme "publiques", on fait donc allusion à un espace de concurrence sociale: pour obtenir une charge, un rôle ou occuper une fonction, il doit y avoir nomination, élection ou cooptation. Voilà le critère qui fonde la démarche. Les fonctions de médecins, notaires et curés sont ainsi exclues de notre corpus, parce qu'elles constituent des charges privées définies par des lois publiques, et que les détenteurs du titre doivent avoir reçu une formation spécifique liée à ces charges.

Cette distanciation par rapport à une définition trop hermétiquement étatique des postes institutionnels trouve sa justification dans les finalités même de notre problématique. En recourant à une telle stratégie, nous voulons répondre à l'appel de Donald Fyson qui soulignait «l'importance d'étudier non seulement les structures gouvernementales sous le contrôle direct de l'administration centrale, mais aussi tous les moyens dont disposent les élites locales pour gouverner les sociétés locales ${ }^{11}$ ». En d'autres termes, nous nous servons du rapport à l'État comme d'un outil pour mieux approfondir notre compréhension de la condition élitaire. Nous ne cherchons pas ici à présenter une vision rétrospective de la construction de l'État capitaliste contemporain.

\section{1 - LE TERRAIN D'ENQUÊte}

Notre terrain d'enquête est la paroisse de Saint-Roch-de-l'Achigan, située au nord-est de l'île de Montréal (voir figure 1). Cette paroisse est détachée de la paroisse-mère de Saint-Pierre-du-Portage-de-L'Assomption en 1787. Les seigneuries de Saint-Sulpice et de L'Assomption connaissent un

11. Donald Fyson, "Les structures étatiques locales à Montréal au début de XIX ${ }^{\mathrm{e}}$ siècle ", Cahiers d'histoire, 17,1-2 (automne 1997): 57. 
développement sans précédent depuis la fin $\mathrm{du}_{\mathrm{XvIII}}^{\mathrm{e}}$ siècle, à l'image de ce qui se produit dans d'autres secteurs de la région de Montréal ${ }^{12}$. En 1831, Saint-Roch compte 2615 habitants répartis en 450 ménages. De la fondation de la paroisse en 1787 jusqu'en 1810, le curé de Saint-Roch dessert un territoire très étendu car, à cette époque, la paroisse comprend tout ce qui s'étend au nord et à l'ouest de son église. Les territoires de Saint-Esprit et de Saint-Lin-de-Lachenaie font alors partie intégrante de Saint-Roch. Saint-Esprit se constitue toutefois en paroisse dès 1810 et SaintLin est délimité comme une région distincte à partir des années $1820^{13}$, même si le détachement officiel ne survient qu'en $1835^{14}$. Notre étude porte toutefois sur le seul secteur de Saint-Roch-de-l'Achigan, moins les deux territoires détachés de cette paroisse avant et après 1810. Pour faciliter ce découpage, nous avons choisi de faire commencer l'étude en 1810, date du détachement de la paroisse Saint-Esprit; elle s'arrête en

\section{FIGURE 1}

\section{Saint-Roch-de-I'Achigan en 1825}

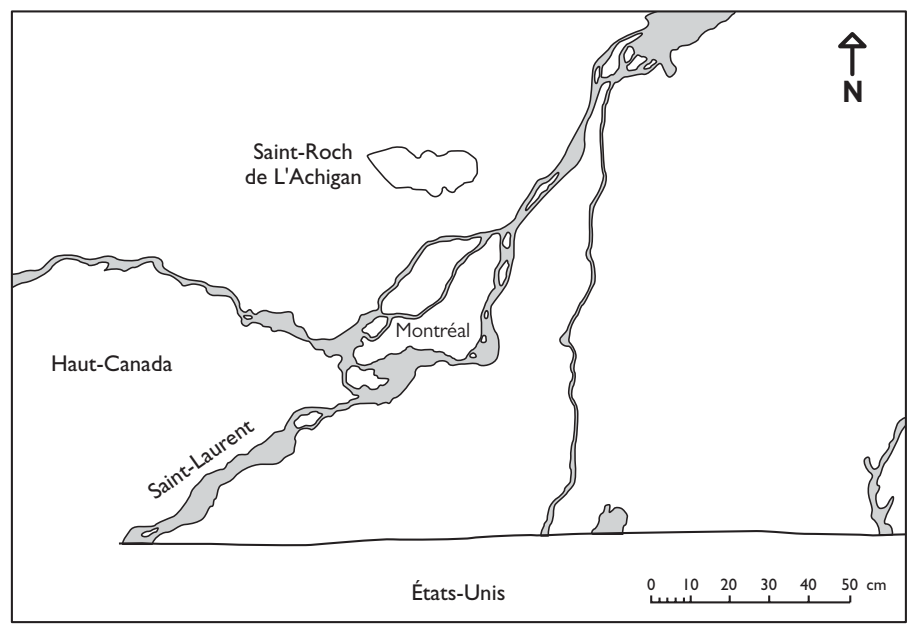

12. Voir entre autres Christian Dessureault, "Crise ou modernisation? La société maskoutaine durant le premier tiers du XIx ${ }^{\mathrm{e}}$ siècle », RHAF, 42,3 (hiver 1989): 359-388.

13. Les actes notariés relatifs à la voirie et le choix des marguilliers dans cette période en sont des indicateurs. En 1831, les recenseurs traitent Saint-Lin comme un territoire distinct de SaintRoch.

14. Les limites de la paroisse sont officialisées par le décret canonique du 12 janvier 1832 et les registres paroissiaux s'ouvrent en 1835. Voir à ce sujet ANQM, fonds E-4: Dossier d'érection de paroisse de Saint-Roch-de-l'Achigan. 
1840, soit au moment de l'Acte d'Union. Ce choix s'explique par la volonté de travailler sur les structures de pouvoir qui précèdent les réformes Durham.

La paroisse de Saint-Roch-de-l'Achigan est plutôt éloignée des grands centres. Au début de notre période d'étude, elle constitue "l'arrièrepays» du bourg de L'Assomption, mais possède néanmoins son propre noyau villageois. Dès 1815 , un certain nombre de marchands, d'artisans et de professionnels s'y activent ${ }^{15}$. Au recensement de 1831, le curé, l'agent seigneurial, le notaire et les marchands habitent tous au village ou à proximité, de même que plus de $30 \%$ des artisans de la paroisse. Ceux-ci représentent alors $12,8 \%$ des chefs de ménage ${ }^{16}$. La majorité des habitants se consacre principalement à la culture du sol: cultivateurs, fermiers et journaliers représentent ensemble $77 \%$ des chefs de ménage à Saint-Roch ${ }^{17}$. Les activités économiques du village apparaissent donc moins comme le germe d'industries rurales autonomes que comme un soutien à l'activité agricole. La forme villageoise saint-rochoise s'apparente à ce que l'historien Serge Courville appelle un «bourg de l'intérieur», c'est-à-dire une agglomération située loin "des grands axes de circulation terrestre [et qui] ont parfois des fonctions plus banales orientées vers la satisfaction des besoins locaux ou sous-régionaux ${ }^{18}$ ".

\section{2 - LES INSTITUTIONS PUBLIQUES}

La vie publique dans la paroisse de Saint-Roch tourne autour d'un certain nombre de charges ou de fonctions. Leur inventaire a d'abord été réalisé à partir de la documentation scientifique. Par la suite, nous avons consulté le Code rural, rédigé par le protonotaire Joseph-François Perrault en 1832, qui donne une vue d'ensemble des postes constituant l'armature du pouvoir local ${ }^{19}$. L'examen des greffes de notaires a finalement

15. On retrouve la mention d'un village à Saint-Roch dans les archives notariées dès le début de notre période d'étude. L'historien Serge Courville relève également la présence d'un village sur le territoire de Saint-Roch dès 1815. Voir Serge Courville, Entre ville et campagne - L'essor du village dans les seigneuries du Bas-Canada (Québec, Les Presses de l’Université Laval, 1990), 274.

16. Cette proportion correspond à ce qui a été mesuré dans la seigneurie de Saint-Hyacinthe, où Dessureault et Legault ont calculé 9,2\% d'artisans en 1831 .

17. À ce titre, cela correspond à l'ordre de grandeur établi par Christian Dessureault et Roch Legault pour la seigneurie de Saint-Hyacinthe en 1831, où la paysannerie compte pour $82,5 \%$ de la population. Voir C. Dessureault et L. Legault, loc. cit., 112.

18. S. Courville, op.cit., 195.

19. Joseph-François Perrault, Code rural à l'usage des habitants tant anciens que nouveaux du BasCanada (Québec, Imprimerie Fréchette, 1832), 31 p. 
permis d'enrichir les informations sur certaines charges publiques moins bien connues $^{20}$. Précisons toutefois que la structure institutionnelle retenue pour la présente analyse ne prétend pas être exhaustive: seules les fonctions et charges occupées par des Saint-Rochois durant la période considérée ont été cataloguées ${ }^{21}$. Nous sommes également conscients que certains rôles et fonctions - notamment ceux d'arbitre et de procureur - sont à la frontière de notre définition de "charge publique », mais nous n'avons pas voulu nous couper de cette réalité. Nous jugeons que ces fonctions peuvent apporter un éclairage différent au sujet des statuts élitaires. Nous les incluons donc dans notre analyse, de manière à mieux délimiter la part de leur rôle, de leur impact et de leur signification au sein de la structure de pouvoir local.

TABLEAU 1

\section{Classification des fonctions et rôles publics exercés à Saint-Roch- de-I'Achigan par niveau institutionnel, I810-1840}

\begin{tabular}{ll}
\hline Institutions & Fonctions et rôles \\
\hline Majeures & Juge de paix \\
& Officier de milice haut gradé (état-major) \\
& Grand juré \\
& Commissaire \\
& Député \\
Médianes & Officier de milice subalterne \\
& Marguillier \\
& Syndic paroissial \\
& Syndic scolaire \\
& Inspecteur des chemins \\
& Huissier \\
& Syndic de district \\
& Sous-voyer \\
Inspecteur des clôtures et fossés \\
Arbitre \\
Crieur \\
Procureur \\
Priseur \\
\end{tabular}

20. Les efforts ont été concentrés sur les notaires résidant à Saint-Roch entre 1810 et 1840 . Il s'agit de Jean-Marie Faribault, Thomas Bédard, François Allard, Jean-Baptiste Archambault, Auguste Rocher et Louis Archambault.

21. Fonctions et charges entre autres absentes de notre analyse : les petits jurés, greffiers des commissaires des petites causes, maîtres de poste. On peut également noter l'absence des sergents de milice et des connétables des églises, faute de documents officiels pour identifier formellement les individus qui occupent ces postes. 
Nous évaluons cet ensemble de fonctions et de rôles, en considérant la portée de chacun sur le plan social et décisionnel. Suivant ce procédé, nous avons élaboré un classement des divers rôles publics locaux dans une logique hiérarchique en les regroupant en trois niveaux: les rôles majeurs, les rôles médians, et les rôles mineurs (tableau 1). Effectivement, l'exercice d'un rôle public peut, d'une part, engendrer un effet sur un petit ou un grand nombre d'individus et, d'autre part, avoir un impact fort ou faible sur le devenir de la communauté. Habituellement, les deux phénomènes vont de pair: l'exercice d'un rôle public, dont les actions interpellent un grand nombre de personnes, comporte nécessairement un impact décisionnel fort pour le devenir de la communauté. Par ailleurs, certaines institutions sont sollicitées de manière ponctuelle, tandis que d'autres agissent de façon permanente sur la vie collective.

\section{Les rôles majeurs}

Les rôles majeurs commandent le rayon d'action le plus large et leurs décisions ont un impact important sur la communauté. Le pouvoir décisionnel lié à ces rôles agit sur un territoire dépassant le cadre paroissial. Ces postes représentent des instances intermédiaires entre les communautés locales et le gouvernement colonial.

La fonction de juge de paix, mentionnée en premier lieu au tableau 1, est l'une des plus importantes dans les communautés locales au BasCanada. Après la Conquête, cet officier public devient un intermédiaire administratif et judiciaire entre l'État et la population. Les responsabilités attachées à ce rôle touchent à la réglementation publique en général: les licences de tavernes, les animaux errants, les cas d'inconduite à l'église et d'ivresse publique sont soumises au regard du juge de paix ${ }^{22}$. Selon J. I. Little, mis à part l'entretien des routes sous la gouverne des Grands Voyers et inspecteurs des chemins, «the formal regulation of public affairs remained largely in the hands of the $[\ldots]$ justices of the peace $^{23} \ldots[\ldots]$ » Donald Fyson trace de son côté un parallèle avec le rôle des officiers de milice: "Under the system established by the 1787 ordinance, militia officers were required to be the main agents of the criminal justice system in the countryside [...] But as before 1787, militia officers were in practice not the main agents of the criminal justice

22. Voir dans Allan Greer, op. cit., 93.

23. J. I. Little, State and Society in Transition - The Politics of Institutional Reform in the Eastern Townships, 1838-1852 (Montréal, McGill-Queen’s University Press, 1997), 3. 
system in the countryside, at least at the level of the justices of the peace $^{24}$ ».

Les juges de paix assument en fait le double côté de la médaille: la prévention des désordres, et la réception des plaintes à la suite d'un méfait ${ }^{25}$. Ils veillent à l'exécution des ordres de la cour et font surveiller les prisonniers dont il faut assurer le déplacement vers la prison la plus proche. Ils remplissent aussi un rôle administratif au niveau local en signant des permis ou en présidant des assemblées officielles. Entre 1803 et 1820 , les juges étaient également aptes à faire prêter serment ${ }^{26}$. En 1830, une loi précise des critères pour accéder à cette charge ; les juges de paix doivent désormais posséder des biens fonciers d'une valeur annuelle de plus de $£ 30$ cours Halifax ${ }^{27}$. Or, les Saint-Rochois possédant des biens fonciers d'une telle valeur sont très peu nombreux.

Les officiers de milice de l'état-major, qui viennent au deuxième rang des rôles majeurs, accaparent également une part importante de l'autorité. Présente depuis longtemps, la milice sédentaire a acquis au fil du temps une légitimité considérable dans le monde rural bas-canadien. Cette institution est d'abord une réserve d'hommes pour les autorités britanniques, en cas de conflits militaires ${ }^{28}$. Dans le cas d'une mobilisation générale, les officiers supérieurs possèdent le plus haut échelon de commandement au niveau régional. Au même titre que les «magistrats de la paix", les officiers supérieurs tiennent donc un rôle s'étendant audelà de leur paroisse.

Dans leur analyse du bataillon de Saint-Hyacinthe entre 1808 et 1830 , Christian Dessureault et Roch Legault établissent une classification des différents postes d'officiers en départageant les membres de l'état-major des officiers subalternes ${ }^{29}$. Nous inspirant de leur classement, nous regroupons dans la catégorie des hauts gradés les charges de lieutenantscolonels, colonels et majors, auxquelles nous avons ajouté, pour les fins de notre étude, les autres membres de l'état-major. Du point de vue militaire, l'officier de l'état-major occupe un niveau hiérarchique plus

24. Donald Fyson, Criminal Justice, Civil Society and the Local State: The Justices of the Peace in the District of Montreal, 1764-1830, op. cit., 243-244.

25. Ibid., 65-66.

26. Ibid., 112-113.

27. En supposant un rendement annuel de l'ordre de $10 \%$ comme l'a fait Donald Fyson, on comprend que le bien-fonds exigé se traduit par la possession d'une terre d'une valeur de $£ 300$ cours Halifax.

28. C. Dessureault et R. Legault, loc. cit., 93.

29. Ibid., 97. 
élevé que les officiers subalternes tels les capitaines. La consultation des greffes notariés nous autorise, par ailleurs, à penser que les responsabilités civiles qui incombent aux capitaines de milice sont également endossées par les officiers de l'état-major. Cela signifie que les responsabilités stipulées dans l'Acte de 1787 reviennent à l'officier supérieur plutôt qu'au capitaine, ce qui n'exclut toutefois pas la participation de celui-ci à certaines interventions d'importance, comme l'identification d'un corps par exemple. Le maintien de l'ordre public n'est pas l'apanage des officiers supérieurs, mais la décision finale leur appartient dans les cas litigieux. De plus, ce sont les officiers supérieurs qui accaparent le rôle principal lors des événements concernant l'ensemble de la paroisse. Par exemple, l'élection des syndics scolaires en 1829, à Saint-Roch, est présidée par l'officier le plus haut gradé ${ }^{30}$. Les capitaines conservent néanmoins des responsabilités importantes dans leurs propres districts à l'intérieur de la paroisse.

La charge d'officier haut gradé est nominative: ce sont les fonctionnaires coloniaux qui distribuent les commissions d'officiers supérieurs. Les critères d'éligibilité sont informels: ce n'est qu'en 1830, à la faveur d'un contexte particulier, que l'on fixe par un article de loi un bien-fonds minimum d'une valeur annuelle de $£ 50$ cours Halifax pour accéder aux postes d'officiers "supérieurs ${ }^{31}$ ».

À la suite des juges de paix et des officiers supérieurs de la milice, les hommes qui siègent à titre de grands jurés dans les tribunaux de Montréal prennent part au niveau institutionnel majeur. Le Grand jury, qui agit aussi comme organe de consultation, apparaît au Canada en 1764. Rouage important du système de justice, la fonction n'a pourtant que très peu retenu l'attention des historiens jusqu'à maintenant. Donald Fyson pousse le plus loin l'exploration de cette institution. Pour décrire le rôle des grands jurys, Fyson utilise entre autres une déclaration du président des Sessions trimestrielles de 1836: «une partie importante de leur charge [consiste] à faire des représentations au gouvernement sur l'administration des affaires locales et publiques $^{32}$ ». Il ajoute plus loin que les grands jurys «sont généralement regardés comme des émanations de l'opinion publique ${ }^{33} »$. Concrètement, cette institution est un

30. Ibid., 95-96.

31. A. Greer, "L'habitant et l’État...», loc. cit., 103.

32. D. Fyson, "Jurys, participation civique et représentation... », loc. cit., 113.

33. Ibid. 
organe de consultation, qui rédige des rapports à la suite de questions soumises par les juges des tribunaux. Plusieurs de ces représentations débouchent sur des recommandations concernant des questions locales, notamment tout ce qui a trait aux places de marché, au patrimoine bâti et au fonctionnement administratif local.

À Saint-Roch, les personnes invitées à siéger comme grand juré le sont toujours à titre ponctuel. En se joignant à cette instance judiciaire, ces hommes donnent une voix à leur communauté locale ${ }^{34}$. Toutefois, l'impact de cette fonction est d'abord dans la relation qu'elle institue entre la communauté locale et l'instance régionale de pouvoir. Cette interaction nourrit l'évolution des affaires locales, en même temps qu'elle modifie chez les élites locales la compréhension de leur système légal. Ceux qui endossent la fonction de grand juré sont donc des véhicules de légitimité de l'administration coloniale, en même temps qu'ils peuvent en être les critiques. Fyson souligne à ce propos que «les représentations des grands jurys sont parfois utilisées pour critiquer l'administration municipale des juges de paix et leurs officiers inférieurs ${ }^{35}$ ».

La charge de grand juré est nominative. Au même titre que les juges de paix et les officiers hauts gradés, les grands jurés doivent satisfaire à des critères d'éligibilité fondés sur la propriété foncière. Pour siéger comme grand juré à la Cour du Banc du Roi, la propriété doit avoir une valeur annuelle de $£ 25$ cours Halifax, alors qu’au niveau des Sessions trimestrielles, on exige une valeur annuelle de $£ 15$ cours Halifax de bienfonds $^{36}$. À partir de ces critères, on met sur pied des listes de jurés potentiels.

Les diverses charges de commissaires appartiennent également aux rôles majeurs. Ces charges sont souvent créées pour répondre à des besoins spécifiques et dans des contextes particuliers. C'est d'abord le cas de la charge de commissaire au serment d'allégeance durant la guerre de 1812. Puis, s'ajoute la charge de commissaire au tribunal des petites causes qui fait son apparition en $1821^{37}$. Ces commissaires ont le pouvoir

34. Ibid., 113-114 et 120. J. I. Little, dans un ouvrage où il décrit le système étatique local, souligne cette contribution des jurés dans le débat public, notamment en ce qui concerne les affaires de voirie. Voir J. I. Little, op.cit., 60-61.

35. D. Fyson, «Jurys, participation civique et représentation...», loc. cit., 117.

36. ANC, RG, 4B19, vol. 19.

37. Cette charge est apparue à la suite d'une loi sur le recouvrement des petites dettes en 1819. Voir Sylvio Normand, "Justice civile et communauté rurale au Québec, 1880-1920», Les Cahiers de Droit, 25,3 (septembre 1984): 582. 
de statuer sur des causes civiles concernant des rentes, des dettes, des salaires et des biens, pour autant que le montant en litige n'excède pas 6 livres 5 sols ancien cours $^{38}$. La paroisse de Saint-Roch accueille un tel tribunal qui demeure actif du 29 janvier 1822 jusqu'au $1^{\text {er }}$ mai $1825^{39}$. En 1832, la charge de commissaire aux chemins vient se substituer à celle d'inspecteur des chemins. Perrault, dans son ouvrage publié l'année même de l'introduction de cette fonction, la décrit ainsi :

[les] commissaires des chemins sont des officiers nouvellement nommés dans chaque paroisse pour exercer les devoirs des Grands Voyers pendant deux ans. Ils peuvent employer un arpenteur pour dresser les plans et procès-verbaux des chemins et ponts à faire ou à changer. Ils font homologuer ces plans et procès-verbaux devant les magistrats du comté, dont copie doit être transmise au Grand Voyer du district. Ils sont autorisés à fixer les frais et dépens et à en faire prélever le montant par saisie et vente, tant contre les propriétaires présents qu'absents. Les inspecteurs et sous-voyers sont tenus d'exécuter leurs ordres, comme ceux des Grands Voyers ${ }^{40}$.

On ne renouvelle pas la loi en 1835 : l'expérience n'aura duré que trois ans.

On possède peu d'informations sur les critères d'éligibilité à ces trois postes de commissaires. Dans le cas du commissaire au tribunal des petites causes, Fyson et J. I. Little signalent le recours aux juges de paix qui connaissent déjà le système de justice et l'administration coloniale ${ }^{41}$. Pour le poste de commissaire aux chemins, la législation mentionne qu'il est élu lors d'une assemblée paroissiale présidée par le juge de paix ou le plus ancien officier de milice de la paroisse ${ }^{42}$.

L'Acte constitutionnel de 1791 amène la création d'une nouvelle charge publique: député. Cette charge politique constitue la seule charge élective parmi les rôles institutionnels majeurs. Le député a l'occasion de participer aux débats sur les questions de l'heure à la Chambre d'Assemblée et peut être appelé à voter sur des projets de lois. Il participe au gouvernement de la colonie, autant en regard des affaires locales, régionales que "nationales» — entendre «coloniales». Sur le plan local, les paroisses qui hébergent un député lui accordent généra-

\footnotetext{
38. J. I. Litle, op. cit., 49 et 52.

39. ANC, RG68, Commissions et lettres patentes, vol. 7, 21 et 64-67.

40. J.-F. Perrault, op. cit., 28.

41. Voir D. Fyson, Criminal Justice, Civil Society..., op. cit., 252; J. I. Little, op. cit., 52.

42. Tel qu'il est énoncé dans un acte passé au Parlement provincial le 25 février 1832. Voir dans les Statuts provinciaux du Bas-Canada, 1792-1836.
} 
lement une place de choix dans les affaires publiques. Ainsi, les principaux projets de la communauté passent souvent entre ses mains. J. I. Little note d'ailleurs le rôle important qu'ils ont joué dans la mise en place des écoles de syndics, agissant entre autres comme inspecteurs ${ }^{43}$. Cette charge politique est en théorie accessible à tous les propriétaires qui possèdent le cens électoral minimum les rendant éligibles à voter ${ }^{44}$. Le cens est fixé à $£ 2$ cours Halifax ${ }^{45}$, un niveau relativement bas. Néanmoins, l'exigence de ce bien-fonds écarte les journaliers et les fermiers, soit $25 \%$ des chefs de ménage de Saint-Roch en 1831.

Les juges de paix, officiers hauts gradés, grands jurés, commissaires et députés remplissent des rôles dans le pouvoir local qui ont une portée extraparoissiale. À ce titre, ils participent tous à faire le pont entre la communauté locale et le monde extérieur.

\section{Les rôles médians}

Les rôles médians présentés au tableau 1 ont aussi un impact sur les structures de l'ensemble de la paroisse. Ces fonctions n'ont toutefois aucune prise à l'extérieur de Saint-Roch. Parmi ces seconds rôles, les officiers de milice subalternes et les marguilliers, parce qu'ils participent à des institutions actives de façon permanente sur le territoire, ont davantage de poids social et de prestige. Les charges de syndic, d'inspecteur des chemins et d'huissier sont également classées parmi les rôles médians, même s'ils sont moins permanents et moins prestigieux.

Les officiers subalternes assurent l'encadrement des milices à l'échelle des districts de chaque paroisse (un ou deux rangs selon le cas). Le premier et le plus connu de ces officiers est le capitaine de milice. Cependant, dans la plupart des districts, il est assisté d'un lieutenant et d'un enseigne. La permanence de cette institution militaire a contribué à créer une image forte du capitaine de milice.

La fonction de capitaine va bien au-delà des pouvoirs finalement assez limités que lui confère la loi. La charge a des racines historiques profondes dans les campagnes canadiennes-françaises; une sorte d'aura

43. J. I. Little, op. cit., 172.

44. Les femmes autant que les hommes, selon l'interprétation de France Galarneau. Voir France Galarneau, L'élection dans le quartier ouest de Montréal en 1832: analyse politico-sociale, mémoire de maîtrise (histoire), Université de Montréal, 1978, 28.

45. Pour des renseignements sur la participation électorale, voir Pierre Tousignant, La genèse et l'avènement de la Constitution de 1791, thèse de doctorat (histoire), Université de Montréal, 1971, 488 p.; voir également Nathalie Picard, Les femmes et le vote au Bas-Canada de 1792 à 1849, mémoire de maîtrise (histoire), Université de Montréal, 1992, 34-45. 
l'enveloppe et les rituels qui l'entourent, tels la cérémonie du mai [...] et le «banc du roi» qui lui est réservé à l'avant de l'église paroissiale, font du capitaine un véritable leader dans sa communauté, en même temps qu'un représentant du roi ${ }^{46}$.

Les capitaines de milice doivent remplir diverses tâches. Au premier chef, il faut mentionner les besognes proprement militaires de ces officiers. Pour Donald Fyson, ces tâches se résument à «l'organisation du rassemblement annuel de la paroisse, l'exécution de la corvée pour le transport des provisions, le cantonnement des troupes, etc. ${ }^{47}$ " Christian Dessureault et Roch Legault insistent pour leur part sur l'éventail des responsabilités civiles du capitaine:

On peut aussi requérir ses services pour le maintien de l'ordre dans la paroisse et, le cas échéant, pour l'identification des causes de décès accidentels ou suspects [...] En 1822, les officiers de milice sont invités à soutenir les efforts du clergé dans la confection d'un recensement [...] les officiers sont amenés à s'impliquer dans le fonctionnement du processus électoral et dans la mise en place de nouvelles instances politiques locales [...] Ainsi, à partir de 1822, les officiers rapporteurs qui ont la responsabilité, dans chacun des comtés, de veiller au bon déroulement de ces élections peuvent désormais employer et commander des officiers de milice ou des officiers de paix pour assurer l'ordre public et l'accès aux bureaux de scrutin à tous les électeurs ${ }^{48}$.

Les capitaines de milice interviennent donc dans plusieurs aspects de la vie publique: les élections, les écoles, la justice, la réglementation, l'ordre public. Tout comme les charges de hauts gradés, celles d'officiers de milice subalternes sont nominatives et distribuées par les officiers supérieurs. À partir de 1830, elles requièrent un bien-fonds inférieur de moitié aux officiers supérieurs, soit $£ 25$ cours Halifax ${ }^{49}$.

La fonction de marguillier bénéficie d'un lustre presque similaire à celui de capitaine de milice. Cette fonction a des racines historiques aussi profondes et elle tire également sa légitimité du fait que la vie religieuse est au centre de la vie communautaire. Le marguillier incarne le lien entre la communauté et l'autorité cléricale dans l'administration matérielle des paroisses ${ }^{50}$. Pour André Boucher, leurs tâches ne se limitent pas

46. A. Greer, loc. cit., 100.

47. D. Fyson, "La paroisse et l'administration étatique... », loc. cit., 33.

48. C. Dessureault et L. Legault, loc. cit., 95-96.

49. A. Greer, loc. cit., 103.

50. Anne Bonzon-Leizerovici, auteure d'un travail portant sur la fabrique en France, postule que les paroissiens assument presque entièrement la partie matérielle de ce culte; au Québec, on 
à «tenir les comptes de la fabrique ». Les marguilliers sont, entre autres, responsables de crier les bancs à l'enchère, d'effectuer la quête de l'Enfant-Jésus, de mettre les terrains de la fabrique en valeur, d'acheter les ornements pour le culte, de planifier les travaux de réparation, d'assurer le bon ordre dans l'église et de surveiller les employés de la fabrique $^{51}$. Enfin, dernière responsabilité, mais non la moindre, les marguilliers ont le pouvoir de recouvrir les dettes de la fabrique. Christian Dessureault et Christine Hudon attestent l'existence de "quatre règles formelles ou tacites " pour accéder à la charge de marguillier:

Seuls les hommes, de préférence les chefs de famille d'âge mûr, peuvent remplir cette fonction. Les candidats au poste de marguillier doivent aussi répondre d'une bonne conduite morale et religieuse. En outre, la fonction est réservée aux propriétaires de biens fonciers résidant dans la paroisse. Enfin, on veille, autant que possible, à établir une rotation entre les rangs, afin que chaque partie de la paroisse soit représentée périodiquement au conseil de la fabrique ${ }^{52}$.

Ces critères sont sensiblement les mêmes pour la charge de syndic paroissial. Individu élu par l'assemblée générale de la paroisse, le syndic est choisi pour assurer la concrétisation de projets spécifiques. Le plus souvent, une paroisse se donne des syndics pour chapeauter la construction d'une église ou d'un presbytère. Les syndics doivent établir les besoins du projet et le mener à terme. Ils devront ainsi

$[\ldots]$ faire un état et estimation des dépenses auxquelles la construction [...] pourra monter, et aussi un acte de répartition ou état de ce que chaque individu possédant des terres ou emplacements dans la dite paroisse sera tenu de payer et de fournir, et à faire les marchés, surveiller l'exécution des travaux ${ }^{53} \ldots$

La charge commande également aux syndics de chaque district de collecter l'argent nécessaire auprès des paroissiens pour la réalisation des travaux.

observe sensiblement la même chose au début du xix ${ }^{e}$ siècle. Voir là-dessus Anne BonzonLeizerovici, "La fabrique, une institution locale originale dans la France de l'Ancien Régime». Historiens-Géographes, 341 (octobre 1993): 273.

51. André Boucher, «Le rôle joué par les marguilliers», dans Pierre Hurtubise, op. cit. 164, 167-168.

52. C. Dessureault et C. Hudon, loc. cit., 419-420.

53. ANQM, J.-B. Archambault, 1er octobre 1837, loc.cit. 
Les syndics scolaires sont eux aussi élus par leur collectivité pour gérer des biens communs. Cette charge apparaît avec la loi sur les écoles d'assemblée votée en $1829^{54}$. Les syndics «se voyaient confier le contrôle, la direction, la régie, le maniement et l'administration exclusive des affaires des écoles ${ }^{55}$ \%. Ils vont ainsi acheter les terrains nécessaires à la construction de nouvelles écoles, faire bâtir ces écoles et engager des maîtres compétents $^{56}$. Ces syndics deviennent en quelque sorte des agents du gouvernement au même titre que les officiers de milice et les juges de paix. Le rôle de syndic scolaire s'exerce à l'intérieur d'un mandat précis, après lequel de nouvelles élections surviennent. À Saint-Roch, la dernière élection connue de syndics scolaires a lieu le 3 septembre $1832^{57}$.

Contrairement aux syndics paroissiaux et scolaires, les inspecteurs des chemins sont régulièrement actifs pendant toute la période à l'étude. Dans le premier tiers du $\mathrm{XIX}^{\mathrm{e}}$ siècle, la voirie occupe une place importante dans les activités publiques de Saint-Roch-de-l'Achigan. Les avancées rapides de la colonisation et l'accélération des échanges sont des facteurs à considérer dans ces développements ${ }^{58}$. L'inspecteur dirige les travaux relatifs aux routes et aux ponts dans sa localité. Un peu à la manière du syndic paroissial, c'est lui qui mène les opérations, à la différence que l'inspecteur supervise les travaux ordonnés par le Grand $V$ oyer $^{59}$. Il détermine les besoins en matériaux et fait dresser un acte de répartition des dépenses. Enfin, il choisit les modalités pour mener le projet à terme, par le recours aux corvées ou à un entrepreneur. Même s'il ne fixe pas les projets à développer en priorité pour sa communauté, l'inspecteur a tout de même une influence sur le rythme et l'efficacité avec lesquels il met en chantier les projets. L'inspecteur des chemins est nommé par le gouvernement colonial pour une durée qui varie selon les années: à Saint-Roch, si certains inspecteurs semblent en poste pendant deux ans, certains n'y restent que six mois. À notre connaissance, aucun

54. C. Dessureault et R. Legault, loc. cit., 95.

55. Andrée Dufour, La scolarisation au Bas-Canada, 1826-1859: une interaction État-communautés locales, thèse de doctorat (Histoire), Université du Québec à Montréal, 1992, 89. Le contenu du texte de loi est en italiques dans le passage cité.

56. Plusieurs transactions notariées relatent la fébrilité qu'a pu susciter l'instauration du régime des syndics. Les greffes de Jean-Baptiste Archambault et Auguste Rocher font état d'achats de terres et de marchés conclus avec des entrepreneurs par les syndics; des demandes de remboursement de frais encourus sont également adressées au gouvernement.

57. ANQM, Auguste Rocher, 3 septembre 1832: Rapport d'élection des syndics.

58. Voir Serge Courville, Entre ville et campagne..., op. cit.

59. D. Fyson, «La paroisse et l’administration étatique...», loc. cit., 33. 
bien-fonds minimal n'est exigé pour être candidat; selon Fyson ${ }^{60}$, les candidats sont choisis "parmi les propriétaires les plus substantiels", c'est-à-dire les mieux nantis.

La charge d'huissier est également classée comme un rôle médian principalement en raison de son rayon d'action et de la gravité de ses interventions. En effet, même s'il est surtout "chargé de l'exécution des ordres des juges civils ${ }^{61} », l^{\prime}$ huissier est mis à profit dans diverses situations de type juridico-légale, que ce soit pour la justice ou les affaires courantes, ce qui l'amène à intervenir dans toute la paroisse. À SaintRoch par exemple, on remarque sa présence comme crieur lors de ventes par adjudication. C'est également lui qui homologue les avis publics donnés par les inspecteurs des clôtures et des fossés ${ }^{62}$. Les huissiers sont nommés par les autorités coloniales. À moins d'un grave manquement, les hommes choisis demeurent en poste jusqu’à leur mort, ou à tout le moins, leur retraite.

Au total, les officiers de milice subalternes, marguilliers, syndics, inspecteurs et huissiers ont un pouvoir d'intervention sur l'ensemble du territoire paroissial.

\section{Les rôles mineurs}

L'action des rôles mineurs se répercute sur un petit nombre de gens, soit quelques ménages, ou sur un territoire qui s'apparente au district ${ }^{63}$. Avec un rayon d'action aussi court, l'exercice de ces fonctions a une résonance limitée sur le devenir de la communauté.

Les syndics de district, les sous-voyers et les inspecteurs des clôtures et fossés sont parmi les rôles publics ayant prise sur l'ensemble du district. La charge de syndic de district est en tout point calquée sur celle de syndic paroissial, à la différence que le syndic de district opère sur un territoire plus restreint.

Les sous-voyers sont également mandatés pour mener à terme des projets bien spécifiques, relatifs à la voirie. Ils sont appelés à répondre de leur projet même quand celui-ci est complété depuis longtemps. Car

60. Ibid.

61. J.-F. Perrault, op, cit., 29.

62. L'huissier signe toujours une déclaration faisant foi des travaux et actions entrepris; cette déclaration signée est normalement annexée à la fin de l'acte. Voir par exemple ANQM, JeanBaptiste Archambault, 12 juillet 1826: Procès-verbal d'un cours d'eau pour Jean-Baptiste Beauchamps et autres.

63. Nous utilisons le terme de district pour designer les parties - ou différentes zones constituant l'ensemble du territoire paroissial. 
même s'ils ne sont que des officiers publics "de district», ils prennent part au processus décisionnel: conjointement avec l'inspecteur des chemins, ils choisissent l'entrepreneur adéquat et décident de la contribution des habitants (corvées, matériaux), ce pourquoi ils demeurent imputables.

Les inspecteurs des clôtures et des fossés sont sollicités par les habitants de leur district pour évaluer des situations problématiques: projet de clôture entre plusieurs voisins, débouche à creuser entre deux terres, etc. Après avoir pris connaissance du problème, l'inspecteur propose des solutions, dresse une liste des frais à être partagés et réunit les deniers nécessaires. J. I. Little souligne que les inspecteurs des clôtures possèdent également des pouvoirs judiciaires mineurs ${ }^{64}$. La loi qui régit les clôtures et fossés leur permet en effet contraindre ceux qui refusent de contribuer financièrement aux travaux à le faire $e^{65}$. La judiciarisation du processus fait donc de l'inspecteur des clôtures et fossés un véritable médiateur local. Nous n'avons pas d'indication claire quant à la durée de ses fonctions, mais tout indique qu'il est élu pour une année. Enfin, les rôles d'arbitre, de crieur, de priseur et de procureur ${ }^{66}$ agissent comme des supports à la trame de la vie quotidienne. Les fonctions régissent davantage le court terme et les rapports entre un nombre réduit de familles ou d'individus.

D'un bout à l'autre de l'échelle, les différents niveaux institutionnels cohabitent et interagissent entre eux. Les individus qui endossent les charges publiques sont au cœur de ce processus d'interaction. Un examen des individus qui gravitent dans cette structure - le personnel institutionnel - peut donner des pistes pour mieux comprendre l'évolution et les changements de cette même structure.

\section{3- PORTRAIT SOCIAL DU PERSONNEL INSTITUTIONNEL LOCAL}

\section{La méthode d'échantillonnage}

Le portrait du personnel des institutions aide à saisir l'articulation de la structure sociale à la structure institutionnelle. Ce portrait a été tracé à partir de multiples sources. D’abord, certains documents de base ont facilité l'identification des individus siégeant à des postes clés, tels les

64. J. I. Little, op. cit.,123.

65. J.-F. Perrault, op. cit., 19-20.

66. Nous avons pris soin de ne retenir que les procureurs qui n'avaient pas de liens de famille au premier degré avec les personnes qu'ils représentent. 
marguilliers, les officiers de milice ou les juges de paix ${ }^{67}$ Ensuite, les inventaires après décès ont servi au relevé des priseurs et des crieurs qui y sont mentionnés pour services rendus. Enfin, une collecte systématique de marqueurs a été réalisée à partir des registres d'état civil, du recensement de 1831 et surtout d'un ratissage méthodique des greffes de notaires ${ }^{68}$. Cette récolte a entre autres dévoilé toute une variété de fonctions publiques moins documentées, telles les syndics paroissiaux, syndics de district, commissaires, huissiers, arbitres, procureurs, inspecteurs des chemins, sous-voyers et inspecteurs des clôtures et fossés ${ }^{69}$.

Notre démarche de dépouillement nous a permis de repérer 258 individus ayant exercé un rôle public sur le territoire de Saint-Roch. Au moment d'occuper leurs fonctions, c'est-à-dire dans l'intervalle 1810 et 1840 , tous habitent cette paroisse. La classification des institutions et rôles publics en trois niveaux - majeur, médian et mineur - a ensuite orienté notre tri parmi les 258 individus relevés. L'occupation de certains rôles mineurs, assimilables aux affaires communes et ayant un rayon d'action très court et un impact moindre sur les affaires de la communauté, comme les postes de crieur, de priseur, d'arbitre et de procureur, ne pouvait en effet à elle seule valider l'entrée d'un individu dans le corpus du personnel institutionnel. Pour être considérés, ces individus devaient aussi avoir exercé un rôle ayant une portée décisionnelle plus importante, gage d'une réelle légitimité sociale. L’occupation de tous les autres rôles publics permettait cependant de se qualifier pour le corpus-témoin. Par contre, l'occupation d'un des quatre rôles communs (crieur, priseur, arbitre ou procureur) à plus de trois reprises permettait également

67. Au chapitre des documents clés, la disponibilité de documents tels les Comptes et délibérations de la fabrique, couvrant toute la période étudiée, aide à établir la liste des marguilliers. Les Rapports des officiers de milice entre 1807 et 1830 permettent de repérer les officiers originaires de Saint-Roch; les journaux tels L'Ami du peuple et La Minerve peuvent aider à compléter la liste des officiers jusqu'en 1840. L'existence du Book of School Trustees énumère les syndics scolaires élus de 1829 à 1832 à Saint-Roch. De plus, il faut mentionner le Dictionnaire des parlementaires - 1792-1992 de Gaston Deschênes, qui donne les noms des députés élus dans les comtés de L'Assomption et de Lachenaie, dans lequel on retrace des individus de Saint-Roch. Également, la thèse réalisée par Donald Fyson sur l'appareil judiciaire donne les noms des juges de paix de la localité jusqu'en 1830. La consultation des Registres des cours des commissaires et des juges de paix pourra finalement permettre de localiser les individus qui ont été appelés à siéger comme grands jurés.

68. Seuls les greffes des notaires ayant exercé sur le territoire de Saint-Roch-de-l'Achigan entre 1810 et 1840 sont considérés.

69. Contrairement aux charges de marguilliers, d'officiers de milice et de députés par exemple, peu d'études historiques ont accordé de l'importance aux fonctions publiques dites «mineures». 
d'accéder à la cohorte du personnel institutionnel. Ce premier tri a éliminé 107 individus du groupe étudié. Les cas d'homonymie, souvent résolus par le recours aux registres d'état civil ou aux actes notariés, n'ont toutefois pu être dénoués dans cinq cas. Ces derniers ont donc été exclus du corpus, de même que certains autres cas problèmes comme ceux d'individus qui n'ont pas été retracés dans les registres d'état civil ou les recensements, ceux qui ont exercé un rôle public sur une zone mal identifiée, ceux qui demeurent à l'extérieur de la paroisse au moment d'occuper une charge, etc. Sept autres candidats ont ainsi dû être écartés. Au bout du compte, on obtient un corpus réduit de 258 à 139 individus. C'est à partir de ce groupe filtré que notre analyse s'effectuera. Ces 139 individus ont exercé un total de 306 charges publiques entre 1810 et 1840.

\section{La répartition socioprofessionnelle des individus en poste}

Des 139 individus qui occupent une charge institutionnelle à Saint-Roch, seuls 12 ont occupé des postes au sein des institutions majeures. La répartition socioprofessionnelle de ces hommes contraste fortement avec celle de l'ensemble des chefs de ménage de la paroisse. Les groupes socioprofessionnels villageois, à savoir les clercs, membres des professions libérales, marchands, militaires, bourgeois, aubergistes, maîtres d'école et artisans, représentent $66,7 \%$ du groupe des majeurs, alors qu'ils constituent $16,4 \%$ des chefs de ménage de la paroisse (voir tableau 2). À eux seuls, ceux que l'on désigne traditionnellement comme les «notables» du village, c'est-à-dire les membres des professions libérales, marchands, militaires et bourgeois, accaparent près de $50 \%$ des places (voir tableau 2.1). Les cultivateurs demeurent un élément important du groupe des «douze» avec un score de 33,3\%, mais leur poids relatif est plus faible que dans l'ensemble paroissial (tableau 2). Notons par ailleurs l'absence des journaliers, fermiers, rentiers et autres groupes marginaux $^{70}$. Comptant pour plus de $30 \%$ de l'ensemble des chefs de ménage en 1831, ils n’ont aucun représentant parmi les rôles institutionnels majeurs.

Soixante-dix personnes ont par ailleurs occupé un poste dans une institution médiane au cours de la période considérée. Les paysans propriétaires dominent nettement ce groupe avec $67,1 \%$ des effectifs. Les artisans et les notables sont beaucoup moins actifs dans cette

70. Veuves, mendiants, métiers divers. 
TABLEAU 2

\section{Répartition socioprofessionnelle comparée du personnel institutionnel local, 1810-1840 (institutions majeures, médianes et mineures)}

\begin{tabular}{|c|c|c|c|c|c|c|c|c|}
\hline \multirow[t]{2}{*}{$\begin{array}{c}\text { Groupes } \\
\text { socioprofessionnels }\end{array}$} & \multicolumn{2}{|c|}{$\begin{array}{c}\text { Nombre total } \\
\text { de ménages à } \\
\text { Saint-Roch au } \\
\text { recensement de } \\
1831\end{array}$} & \multicolumn{2}{|c|}{$\begin{array}{c}\text { Personnel des } \\
\text { institutions mineures, } \\
1810-1840^{*}\end{array}$} & \multicolumn{2}{|c|}{$\begin{array}{c}\text { Personnel des } \\
\text { institutions médianes, } \\
1810-1840^{*}\end{array}$} & \multicolumn{2}{|c|}{$\begin{array}{c}\text { Personnel des } \\
\text { institutions majeures, } \\
1810-1840^{*}\end{array}$} \\
\hline & Nombre & $\%$ & Nombre & $\%$ & Nombre & $\%$ & Nombre & $\%$ \\
\hline Villageois & 74 & 16,4 & 21 & 18,4 & 17 & 24,3 & 8 & 66,7 \\
\hline Clerc & I & & 0 & & 2 & & 0 & \\
\hline Professions libérales & I & & I & & 2 & & I & \\
\hline Marchands & 3 & & 2 & & 3 & & I & \\
\hline Militaire & I & & I & & I & & I & \\
\hline Bourgeois & 4 & & 3 & & 3 & & 3 & \\
\hline Aubergistes & 4 & & I & & 0 & & 0 & \\
\hline Maître d'école & 5 & & 0 & & 0 & & 0 & \\
\hline Artisans & 55 & & 13 & & 6 & & 2 & \\
\hline Cultivateurs & 233 & 51,8 & 87 & 76,3 & 47 & 67,1 & 4 & 33,3 \\
\hline $\begin{array}{l}\text { Journaliers, } \\
\text { fermiers et autres }\end{array}$ & s 143 & 31,8 & 6 & 5,3 & 6 & $8,6 * *$ & 0 & 0,0 \\
\hline Fermiers & 20 & & 0 & & 0 & & 0 & \\
\hline Journaliers & 92 & & 2 & & 0 & & 0 & \\
\hline Rentiers & 18 & & I & & I & & 0 & \\
\hline Veuves & 12 & & 0 & & 0 & & 0 & \\
\hline Mendiant & I & & 0 & & 0 & & 0 & \\
\hline Pas de mention & 0 & & 3 & & 5 & & 0 & \\
\hline Total & 450 & $100 \%$ & 114 & $100 \%$ & 70 & $100 \%$ & 12 & $100 \%$ \\
\hline
\end{tabular}

* Les mentions socioprofessionnelles du recensement de 1831 ont été utilisées; le cas échéant, les greffes notariés et registres d'état civil ont été mis à profit.

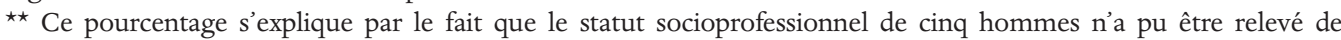
manière formelle. Divers indices nous laissent toutefois croire qu'ils appartiennent à la classe des propriétaires. 
TABLEAU 2.1

\section{Comparaison du personnel institutionnel des sous-groupes villageois} par rapport aux autres ensembles socioprofessionnels

\begin{tabular}{|c|c|c|c|c|c|c|}
\hline \multirow[t]{2}{*}{$\begin{array}{c}\text { Groupes } \\
\text { socioprofessionnels }\end{array}$} & \multicolumn{2}{|c|}{$\begin{array}{c}\text { Personnel des } \\
\text { institutions mineures, } \\
1810-1840^{*}\end{array}$} & \multicolumn{2}{|c|}{$\begin{array}{c}\text { Personnel des } \\
\text { institutions médianes, } \\
1810-1840^{*}\end{array}$} & \multicolumn{2}{|c|}{$\begin{array}{c}\text { Personnel des } \\
\text { institutions majeures, } \\
1810-1840^{*}\end{array}$} \\
\hline & Nombre & $\%$ & Nombre & $\%$ & Nombre & $\%$ \\
\hline Villageois & 21 & 18,4 & 17 & 24,3 & 8 & 66,7 \\
\hline Notables & 7 & 6,1 & 11 & 15,7 & 6 & 50 \\
\hline Clerc & 0 & & 2 & & 0 & \\
\hline Professions libérales & 1 & & 2 & & 1 & \\
\hline Marchands & 2 & & 3 & & I & \\
\hline Militaire & I & & I & & I & \\
\hline Bourgeois & 3 & & 3 & & 3 & \\
\hline Autres & 14 & 12,3 & 6 & 8,6 & 2 & 16,7 \\
\hline Aubergistes & 1 & & 0 & & 0 & \\
\hline Maître d'école & 0 & & 0 & & 0 & \\
\hline Artisans & 13 & & 6 & & 2 & \\
\hline Cultivateurs & 87 & 76,3 & 47 & 67,1 & 4 & 33,3 \\
\hline \multicolumn{7}{|l|}{ Journaliers, } \\
\hline fermiers et autres & 6 & 5,3 & 6 & $8,6 *$ & 0 & 0 \\
\hline Total & 114 & $100 \%$ & 70 & $100 \%$ & 12 & $100 \%$ \\
\hline
\end{tabular}

* Ce pourcentage s'explique par le fait que le statut socioprofessionnel de cinq hommes n'a pu être relevé de manière formelle. Divers indices nous laissent toutefois croire qu'ils appartiennent à la classe des propriétaires.

seconde catégorie. L'exercice de fonctions médianes et majeures semble néanmoins lié au statut de propriétaire foncier.

Au bas de la hiérarchie institutionnelle, 114 individus ont exercé des rôles mineurs. La proportion des cultivateurs augmente encore à ce niveau au détriment des éléments villageois, alors que les journaliers font même leur apparition dans ces rôles publics, avec toutefois un maigre $2 \%$ des effectifs totaux de ce groupe.

Le poids des cultivateurs augmente donc au fur et à mesure que l'on descend les échelons institutionnels: de $33,3 \%$ au sein des institutions majeures, ils montent à $67,1 \%$ chez les médianes pour finir à $76,3 \%$ 
dans les institutions mineures. Inversement, le poids des groupes socioprofessionnels villageois, et surtout celui des notables, s'accroît lorsqu'on grimpe dans l'échelle institutionnelle. En effet, les notables villageois comptent seulement pour 6,1\% des participants aux rôles mineurs, environ $15,7 \%$ de ceux qui occupent des rôles médians et $50 \%$ des hommes qui exercent des rôles majeurs (tableau 2.1).

\section{Les niveaux de richesse des hommes en poste}

Pour mesurer le niveau de richesse des hommes qui participent au pouvoir institutionnel, tous les inventaires après décès rédigés sur le territoire de Saint-Roch-de-l'Achigan ont été compilés. La banque de données qui en résulte permet de positionner notre corpus par rapport à son milieu. Du groupe des majeurs, nous ne possédons que deux inventaires: ceux d'un notaire et d'un cultivateur. Compte tenu du nombre réduit de cas, les données de ces inventaires ne sont pas intégrées au tableau 3. Il est toutefois à propos de noter que la moyenne d'actifs mobiliers de ces deux inventaires se chiffre à quelques 4500 livres, ce qui est bien au-delà des 1200 livres de moyenne pour l'ensemble paroissial.

TABLEAU 3

Comparaison des actifs mobiliers, créances et dettes des chefs de ménage de la paroisse de Saint-Roch-de-l'Achigan et du personnel institutionnel local, I810-1840

\begin{tabular}{lccc}
\hline Catégorie de biens & $\begin{array}{c}\text { Chefs de ménage } \\
\text { (140 cas) } \\
\text { en livres ancien cours }\end{array}$ & $\begin{array}{c}\text { Personnel des } \\
\text { institutions } \\
\text { mineures } \\
\mathbf{( 2 7} \text { cas) } \\
\text { en livres } \\
\text { ancien cours }\end{array}$ & $\begin{array}{c}\text { Personnel des } \\
\text { institutions } \\
\text { médianes } \\
\mathbf{( 1 7} \text { cas) } \\
\text { en livres } \\
\text { ancien cours }\end{array}$ \\
\hline I. Actifs mobiliers & 1192,3 & $17 \mid 4,2$ & 2016,2 \\
I.I Biens de consommation & 298,8 & 370,2 & 489,4 \\
I.2 Biens de production & 111,6 & 142,5 & 177,0 \\
I.3 Cheptel & 397,5 & 557,8 & 636,9 \\
I.4 Stocks & 335,2 & 557,4 & 571,6 \\
I.5 Numéraire & 46,3 & 86,1 & 140,1 \\
2. Créances & 316,6 & 514,5 & 1163,9 \\
3- Dettes & 994,2 & 1085,1 & 675,7 \\
\hline
\end{tabular}


Parmi ceux qui exercent des rôles médians, la distribution des inventaires en fonction des actifs mobiliers laisse voir des écarts substantiels avec l'ensemble des chefs de ménage de la paroisse. Le tableau 3, en effet, montre que la somme totale de leurs actifs mobiliers supplante de $40 \%$ celle de l'ensemble des chefs de ménage de la paroisse. En ce qui concerne les individus exerçant des rôles mineurs, la ventilation de leurs actifs mobiliers laisse voir un écart de l'ordre de $30 \%$ avec la valeur des possessions de l'ensemble des chefs de la paroisse de Saint-Roch. Cet écart est attribuable à l'absence, dans les structures institutionnelles, de la quasi-totalité des individus qui ne sont pas "francs-tenanciers» dans la paroisse. Dans le cas du personnel des institutions majeures, à défaut d'inventaires après décès, il est possible d'avoir un indicateur de richesse en examinant la superficie des terres possédées par ces individus en 1831 (voir tableau 4). Toutes catégories socioprofessionnelles confondues, les 12 hommes qui occupent des charges majeures détiennent en moyenne 227,1 arpents de superficie, alors que seulement $20 \%$ de la population de l'ensemble de la paroisse affiche des avoirs fonciers au-dessus de 100 arpents.

Par ailleurs, la comparaison des avoirs fonciers des cultivateurs qui participent aux institutions médianes et mineures avec l'ensemble des ménages paysans de la paroisse précise la nature de l'articulation des structures institutionnelles aux structures sociales paroissiales. En effet,

TABLEAU 4

\section{Comparaison de la distribution de la superficie des terres possédées par l'ensemble des chefs de ménage et par le personnel des institutions majeures au recensement de I83 I}

\begin{tabular}{lcccc}
\hline $\begin{array}{l}\text { Superficie des terres } \\
\text { possédées (arpents) }\end{array}$ & \multicolumn{2}{c}{$\begin{array}{c}\text { Chefs de ménage } \\
\text { Saint-Roch, 1831 }\end{array}$} & \multicolumn{2}{c}{$\begin{array}{c}\text { Personnel des institutions } \\
\text { majeures, 1831* }\end{array}$} \\
\hline & Nombre & $\%$ & Nombre & $\%$ \\
\hline Moins de 60 & 253 & 56 & 0 & 0 \\
60 à 99 & 109 & 24 & 1 & 8 \\
100 à 199 & 67 & 15 & 5 & 50 \\
Plus de 200 & 21 & 5 & 4 & 42 \\
Total & 450 & 100 & 10 & 100 \\
\hline
\end{tabular}

* Deux des hommes qui participent aux institutions majeures n’apparaissent pas au recensement de 1831. 


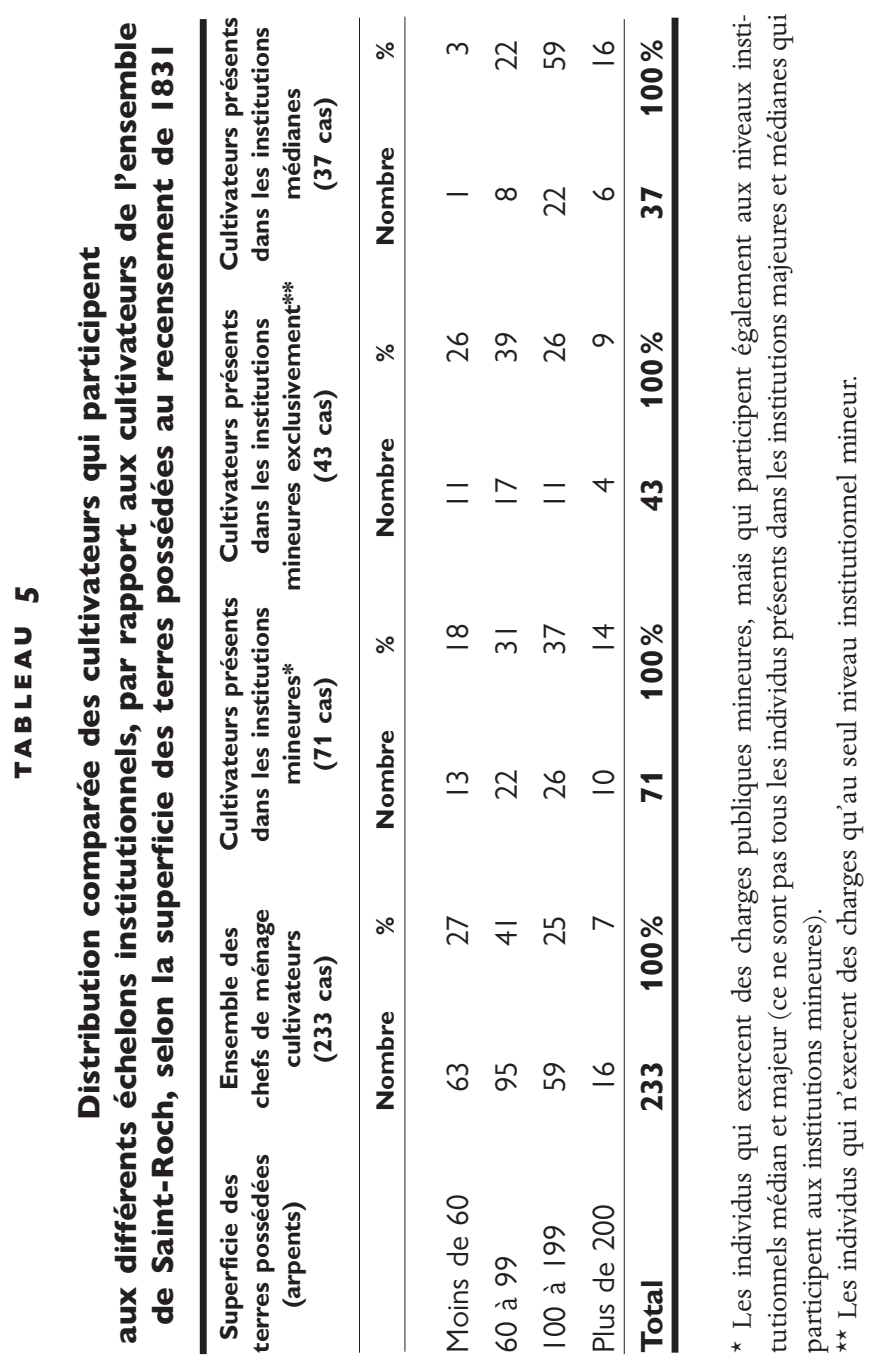


certaines catégories de cultivateurs sont mieux représentées que d'autres à l'intérieur de l'appareil de pouvoir local. Par exemple, nous avons pu repérer 37 des 47 cultivateurs qui participent aux institutions médianes au recensement de 1831, soit plus de $75 \%$ d'entre eux. La distribution de ces cultivateurs en fonction de la superficie des terres possédées laisse voir qu'ils sont parmi les propriétaires les plus importants. Le tableau 5 montre que $75 \%$ des cultivateurs engagés dans les institutions médianes possèdent au-delà de 100 arpents de superficie, contre seulement $32 \%$ chez l'ensemble des cultivateurs de Saint-Roch.

Dans le cas des 114 hommes occupant des postes mineurs, 87 sont cultivateurs. De ces 87 hommes, 71 ont été repérés au recensement. Leur distribution, en fonction du niveau de leurs avoirs fonciers, révèle un écart entre les hommes qui ne participent qu'au niveau institutionnel mineur et ceux qui se retrouvent à la fois dans les niveaux mineurs et supérieurs (médian et majeur). Au total, on constate qu'à mesure que l'on grimpe dans l'échelle institutionnelle, la portion de ceux qui détiennent plus de 100 arpents monte continuellement (tableau 5).

En bout de parcours, la mise en lumière des niveaux de richesse et des statuts socioprofessionnels du personnel des institutions publiques de Saint-Roch conduit à une image hiérarchique. Dans une certaine mesure, les niveaux institutionnels sont représentatifs de la structure sociale de la paroisse, au même titre que les statuts socioprofessionnels le sont d'un certain niveau de richesse ${ }^{71}$. Au sommet, les postes publics renfermant le plus de pouvoir exécutif, avec aux commandes les hommes bénéficiant des statuts socio-économiques les plus élevés: l'agent seigneurial, les marchands, le notaire, les bourgeois ou gros propriétaires fonciers. Au second niveau, se retrouvent les institutions ayant un pouvoir sur l'ensemble paroissial, avec à leur tête des individus issus des strates paysannes de niveau moyen à élevé et quelques artisans fortunés, auxquels s'ajoutent des éléments qui participent également au niveau institutionnel majeur. Les institutions micro-locales, qui servent à régenter les affaires communes relatives aux districts, ferment la marche. Celles-ci sont occupées par des hommes venant de toutes les couches paysannes, les journaliers étant toutefois marginalisés. Les artisans de toutes provenances et une partie des hommes des niveaux majeur et médian complètent cette cohorte. La tendance générale nous conduit

71. La concordance entre les statuts socioprofessionnels et les niveaux de richesse a également été constatée par Christian Dessureault dans le région de Saint-Hyacinthe. Voir à ce sujet C. Dessureault, "Crise ou modernisation?... », loc. cit., 387. 
donc à remarquer que les lieux de concentration du pouvoir décisionnel entraînent à leur suite une concentration des acteurs socio-économiques les mieux outillés.

\section{4 - Liens de parenté et réseauX sociauX}

$\mathrm{Si}$ les niveaux de richesse et les statuts socioprofessionnels participent à déterminer le recrutement des hommes qui gravitent au sein des institutions, il importe de saisir dans quelle mesure les liens sociaux des individus peuvent également avoir un impact sur leur participation aux institutions locales. Cette approche vise d'une part à jauger la densité de ces liens à l'intérieur de chacune des institutions, mais également d'une institution à l'autre, pour enfin déboucher sur les liens entre les niveaux institutionnels à l'échelle de la paroisse entière.

La famille est un point de départ important pour tous les historiens de la société préindustrielle qui s'intéressent à la dynamique sociale ${ }^{72}$. L'étude des liens de parenté permet entre autres d'aborder les questions de la reproduction sociale et de la transmission de biens. Ces liens peuvent également aider à comprendre la dynamique institutionnelle locale.

Les liens de parenté contribuent à modeler le recrutement des institutions publiques de la paroisse. Pour mesurer l'impact des réseaux familiaux dans l'accès aux rôles publics, nous avons répertorié les noms des parents et grands-parents des 139 hommes qui constituent notre cohorte, en plus de ceux de leurs conjointes ${ }^{73}$. Les résultats ont ensuite été intégrés au logiciel généalogique Reunion, ce qui nous a permis de connaître le nombre de liens qui unissent chacune de ces personnes, autant par la parenté de l'homme que de la femme ${ }^{74}$. En tenant compte des remariages, la démarche nous a conduit à effectuer la généalogie courte de 304 individus. Seulement 15 de ces filiations n'ont pu être reconstitués que partiellement ${ }^{75}$.

72. Christian Dessureault est le dernier à l'avoir réaffirmé dans un récent article sur la question des liens de parenté en milieu rural. Voir C. Dessureault, "Parenté et stratification sociale dans une paroisse rurale de la vallée du Saint-Laurent au milieu du xix ${ }^{\mathrm{e}}$ siècle ", RHAF, 54,3 (hiver 2001): 413.

73. Pour reconstituer les lignées familiales, nous avons utilisé la banque numérisée du Programme de recherche en démographie historique (PRDH), les répertoires généalogiques régionaux et locaux ainsi que les registres d'état civil.

74. Pour notre analyse, nous avons considéré autant les liens de parenté proche (parents, frères, beaux-frères, enfants et petits-enfants, grands-parents) que les liens éloignés (oncles, cousins, et neveux).

75. Sept cas concernent des hommes, huit cas des femmes. 
TABLEAU 6

\section{Nombre de liens de parenté selon le niveau institutionnel occupé}

\begin{tabular}{lccc}
\hline & $\begin{array}{c}\text { Nombre de liens } \\
\text { de parenté moyen }\end{array}$ & $\begin{array}{c}\text { Parents } \\
\text { proches* }\end{array}$ & $\begin{array}{c}\text { Parents } \\
\text { éloignés** }\end{array}$ \\
\hline Personnel des institutions mineures & 6,7 & 1,9 & 5,0 \\
Personnel des institutions médianes & 8,7 & 2,6 & 6,4 \\
Personnel des institutions majeures & 9,7 & 4,2 & 5,6 \\
\hline
\end{tabular}

* Parents proches: parenté directe ou par alliances matrimoniales, petits-enfants, enfants, grandsparents, frères, beaux-frères du chef de ménage et de sa conjointe.

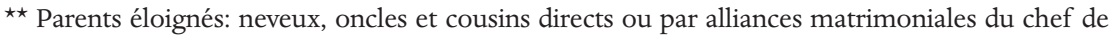
ménage et de sa conjointe.

Dans notre corpus de 139 hommes, c'est à l'intérieur du groupe des 12 individus qui se retrouvent dans les institutions majeures que l'on enregistre la plus haute moyenne de liens de parenté (voir tableau 6) ${ }^{76}$. Toutefois, la distribution inégale des liens de parenté chez ces hommes suggère que ceux-ci sont moins essentiels à l'accession aux postes décisionnels majeurs ${ }^{77}$. Le nombre de liens de parenté à l'intérieur de l'appareil institutionnel ne participe que partiellement à définir l'accessibilité aux charges de haut niveau. Il reste que les individus qui accaparent les rôles publics qui ont le plus de substance au niveau local — tant par la durée que par la nature du pouvoir — à défaut d'avoir un grand nombre de liens, ont tous des liens de parenté proche dans le corpus (frère, beau-frère, beau-père, père, grand-père). Le tableau 6 montre en effet que de façon générale, plus un individu occupe des postes publics importants dans l'appareil de pouvoir local, plus il a de chances de posséder des liens de parenté proche dans le groupe des 139 hommes qui participent au pouvoir institutionnel.

Parallèlement, on constate que le nombre de liens de parenté diminue à mesure que l'on descend les niveaux institutionnels. Ainsi, l'ensemble des hommes qui exercent des rôles majeurs compte en moyenne

76. Dans ce calcul, nous avons pris soin de ne pas compter deux fois les liens qui se répètent chez les deux conjoints d'un même couple.

77. Pour cet aspect particulier, voir le chapitre 4 de notre mémoire de maitrise, aux pages 115 à 117. Un tableau montre le détail des liens de parenté pour chacun des douze individus du groupe. On peut y voir que quatre individus ont vingt liens de parenté et plus, alors que huit autres n'entretiennent des liens de famille qu'avec quatre personnes ou moins. 
TABLEAU 7

\section{Liens de parenté et superficie des terres possédées du personnel institutionnel}

\begin{tabular}{lcc}
\hline & Liens de parenté & $\begin{array}{c}\text { Superficie moyenne } \\
\text { d'arpents possédés en 1831* }\end{array}$ \\
\hline $\begin{array}{l}\text { Personnel des institutions } \\
\text { mineures exclusivement }\end{array}$ & 5,1 & 87,3 (50 cas) \\
$\begin{array}{l}\text { Personnel des institutions } \\
\text { mineures (Ceux participant }\end{array}$ & & 119,5 (88 cas) \\
$\begin{array}{l}\text { aux médianes et majeures compris) } \\
\text { Personnel des institutions médianes }\end{array}$ & 6,7 & 141,5 (77 cas) \\
$\begin{array}{l}\text { Personnel des institutions médianes } \\
\text { (ceux qui participent aux majeures compris) }\end{array}$ & 8,7 & 158,6 (50 cas) \\
$\begin{array}{l}\text { Personnel des institutions majeures } \\
\text { P }\end{array}$ & 9,7 & 227,1 (I0 cas) \\
\hline
\end{tabular}

* Tous groupes socioprofessionnels confondus.

9,7 liens à l'intérieur du groupe institutionnel, contre 8,7 pour ceux qui participent aux institutions médianes et 6,7 pour ceux qui se retrouvent aux institutions mineures. Bien plus, comme le démontre le tableau $7, \mathrm{si}$ l'on retient les individus qui exercent exclusivement des rôles mineurs, en mettant donc de côté ceux qui participent en même temps aux niveaux majeur et médian, le chiffre descend à 5,1 liens de parenté. Voilà qui est presque deux fois moins que ce qu'enregistrent les individus des institutions majeures. La corrélation entre le niveau institutionnel occupé et l'importance des liens de parenté dans notre corpus de 139 hommes ressort ici.

Comme le montre également le tableau 7 , il existe une relation étroite entre le nombre de liens de parenté des individus qui occupent une charge institutionnelle et leurs niveaux de richesse mesurés précédemment. Plus un individu participe à un niveau institutionnel élevé, plus il risque de posséder à la fois plusieurs liens de parenté dans le groupe et des actifs immobiliers importants.

Toutefois, les liens de parenté ne peuvent suffire à définir les pourtours de la vie institutionnelle locale. D'autres liens sociaux, de voisinage, d'affaires ou d'amitié, contribuent à étoffer le portrait social des institutions publiques. Entremêlés aux liens familiaux, ils complexifient la dynamique sociale. Aussi, l'identification d'un certain nombre de réseaux sociaux et de leurs modes de construction nous permet d'appré- 
hender la vie institutionnelle avec plus d'acuité. En effet, la compréhension des intérêts et des finalités qui émanent de ces groupes informels est importante, dans la mesure où ces motivations sont susceptibles d'influencer le cours des affaires publiques locales. Cette façon d'aborder l'étude du pouvoir local invite à s'attarder à l'ensemble des liens sociaux qu'un individu entretient dans son milieu de vie. Ce milieu de vie est représenté par le district, unité géographique de base de la paroisse. L'examen de la densité des liens entre certaines familles ou groupes de familles localisés dans ces districts suggère précisément la présence d'une dynamique de différenciation sociale. Certaines familles se regroupent en fonction de leur notoriété ou de leur réussite économique - mesurable par le niveau de richesse. Ce processus spécifique débouche sur la constitution d'un certain nombre de réseaux sociaux ou de sociabilité. La participation aux affaires publiques est variable d'un réseau à l'autre. Par réseaux sociaux, nous entendons différents ensembles de familles, à l'intérieur desquelles se nouent des sociabilités particulières. Ainsi, nos recherches aboutissent à classer les familles de la paroisse de Saint-Roch-de-l'Achigan en trois réseaux de sociabilité ${ }^{78}$.

En premier lieu, on retrouve le réseau des «notables", qui correspond à ceux que l'on décrit traditionnellement comme les «notables» du village. Le quotidien de ces individus se déroule au village ou tout près: ce sont des bourgeois, des marchands, des notaires ou des médecins. Ils entretiennent également de forts liens avec la notabilité de L'Assomption et le groupe des professions libérales en général. Plusieurs d’entre eux sont des propriétaires fonciers importants; ils savent aussi lire et écrire.

En second lieu vient le réseau des "enracinés», dont la plupart des familles sont rattachées à la terre. Une certaine notoriété et un niveau de richesse souvent supérieur à la moyenne paysanne distinguent ces familles du reste de la paysannerie et les relient entre elles. Les artisans les plus fortunés participent également à ce réseau. Comme plusieurs familles de cultivateurs du groupe, ils font partie des familles enracinées à Saint-Roch depuis la fondation de la paroisse.

78. Cette classification a été réalisée dans une perspective microhistorique, telle qu'elle est définie par l'historien Jacques Revel comme «un ensemble d'interrelations mouvantes à l'intérieur de configurations en constante adaptation». Suivant cette définition, la microhistoire permet également de "repérer les structures invisibles selon lesquelles [le] vécu est articulé». Voir Jacques Revel, "L'histoire au ras du sol», dans Giovanni Levi, dir., Le pouvoir au village: histoire d'un exorciste dans le Piedmont du XVII siècle (Paris, Gallimard, 1989), xii-xiii. 
En troisième lieu, le réseau des "atomisés» - ou des marginaux ferme la marche. Ils demeurent dans les zones moins favorisées de la paroisse, ou encore sont des nouveaux arrivants ou des marginaux. Petits, moyens et certains gros propriétaires, journaliers et artisans cohabitent dans ce groupe, qui se présente davantage comme un ensemble morcelé de plusieurs dizaines de réseaux. Des réseaux dont la sociabilité est davantage repliée sur la famille immédiate, sinon raccrochée à des groupes sociaux moins fortunés ou prestigieux ${ }^{79}$.

L'appartenance socioprofessionnelle et la fortune jouent beaucoup dans ce portrait, mais aussi l'appartenance ou non à un réseau familial bien implanté dans la paroisse. En bout de ligne, on note une certaine adéquation entre les liens de parenté et les réseaux sociaux, les seconds se construisant souvent à partir des premiers.

\section{Des liens sociaux aux cumuls de charges}

La prise en compte de toutes les variables dans le portrait social des hommes qui gravitent au sein de la structure institutionnelle permet d'évaluer avec plus d'acuité l'identité des individus qui cumulent les charges et qui bénéficient donc d'un statut particulier au sein de la communauté. Le phénomène des cumuls témoigne de l'émergence d'une certaine élite institutionnelle à Saint-Roch-de-l'Achigan. Par «élite institutionnelle», nous entendons les individus qui cumulent des postes publics à l'intérieur d'un territoire local - la paroisse dans ce cas spécifique - et qui en retirent du prestige social et du pouvoir vis-à-vis de leurs concitoyens.

Au premier chef, on constate que les cumuls surviennent principalement au sein des réseaux sociaux des "notables» et des "enracinés », dont les membres participent aux niveaux institutionnels majeurs et médians (voir tableau 8).

Ensuite, à l'intérieur de ces deux réseaux sociaux, on constate une concentration de cumuls de charges à l'intérieur de certains groupes ou clans familiaux, tels les familles Archambault et Chaput par exemple. En bout de course, certains des individus enregistrant les cumuls de charges les plus importants endossent aussi certaines caractéristiques. Le statut socioprofessionnel, le niveau de richesse, les liens familiaux, les réseaux de sociabilité et la notoriété constituent autant d'éléments qui compo-

79. Ces réseaux n'ont pas la prétention d'être étanches. Cette classification vise simplement à situer les familles de notre échantillon d'élites à l'intérieur d'un cadre social plus large que celui des élites elle-mêmes. Une étude plus fouillée de la sociabilité rurale locale reste donc à faire. 
TABLEAU 8

Individus ayant occupé le plus grand nombre de charges ou fonctions publiques à Saint-Roch-de-I'Achigan, I 8 I 0-I840

\begin{tabular}{|c|c|c|c|c|c|c|c|}
\hline \multirow[t]{2}{*}{ Personnel institutionnel } & Profession, 1831 & \multicolumn{4}{|c|}{$\begin{array}{l}\text { Nb de charges et/ou } \\
\text { fonctions publiques } \\
\text { occupées, 1810-1840 }\end{array}$} & \multirow[t]{2}{*}{$\begin{array}{c}\text { Actifs } \\
\text { immobiliers } \\
1831 \\
\text { (superficie } \\
\text { possédée) }\end{array}$} & $\begin{array}{c}\text { Nb de liens } \\
\text { s,de parenté } \\
1810- \\
1840\end{array}$ \\
\hline & & naj.** & néd. & $\min .^{*}$ & Total & & \\
\hline Archambault, Jacques père & bourgeois & 4 & 3 & 4 & II & 140 & 26 \\
\hline Archambault, François & marchand & 2 & 2 & 3 & 7 & 294 & 4 \\
\hline Chaput, Pierre & cultivateur & I & 3 & 3 & 7 & 189 & 20 \\
\hline Courteau, Charles & bourgeois & I & 3 & 3 & 7 & 550 & 2 \\
\hline Vinet dit Souligny, Ambroise & cultivateur & 0 & 4 & 2 & 6 & 120 & 5 \\
\hline Archambault, Jacques fils & cultivateur & 0 & 3 & 3 & 6 & 170 & 28 \\
\hline Rocher, Barthelémy & bourgeois & 3 & 1 & 1 & 5 & 213 & 2 \\
\hline Beauchamps, Pierre & cultivateur & 0 & 3 & 2 & 5 & 120 & 6 \\
\hline Gareau, Thomas & cultivateur & 0 & 3 & 2 & 5 & 90 & 9 \\
\hline Dufresne, Jean-Olivier & forgeron & I & 2 & 2 & 5 & 66 & 4 \\
\hline
\end{tabular}

* Institutions majeures, médianes et mineures.

sent le portrait d'ensemble de ces «hommes de pouvoir». En somme, différentes identités sociales cohabitent au sein de la structure institutionnelle. Les multiples mécanismes à l'œuvre dans la création de ces images sociales se traduisent par un ensemble de mouvements reproduits à la figure 2 .

À ce titre, on peut citer l'exemple des Jacques Archambault, Ambroise Vinet dit Souligny et Pierre Chaput. Bien qu'appartenant au groupe des cinq hommes qui exercent les cumuls de charges les plus importants à Saint-Roch entre 1810 et 1840 (tableau 8), aucun d'eux ne figure dans le club sélect des 20 propriétaires fonciers qui possèdent les plus grandes superficies de la paroisse en 1831. De plus, plutôt que d'appartenir au groupe des professions libérales ou à la classe marchande, ces hommes sont issus du monde paysan ${ }^{80}$. C'est à ce niveau qu'intervient le facteur de la notoriété, porte d'entrée du groupe élitaire.

80. Dans le cas de Jacques Archambault, il sera longtemps présenté comme cultivateur avant d'endosser l'épithète de bourgeois. 


\section{FIGURE 2}

\section{Les facteurs de modélisation de l'appareil institutionnel local}

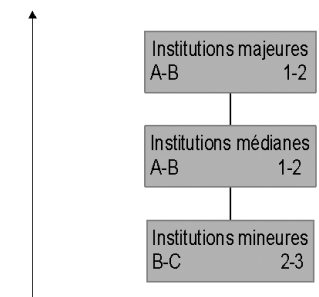

Légende: Statuts socioprofessionnels

Légende: Réseaux de sociabilité

A- Notables villageois

B- Cultivateurs et artisans des strates

1- Notables villageois

2- Enracinés

moyennes et hautes

3- Marginaux et atomisés

C- Cultivateurs et artisans des strates moyennes et basses

\section{CONCLUSION}

$\mathrm{Au}$ point de départ de cette exploration des formes institutionnelles d'avant 1840 , nous avions identifié cinq pistes susceptibles de fournir des éclairages nouveaux. L'examen des structures institutionnelles a en effet permis de confirmer l'existence d'une hiérarchisation des fonctions (piste 1), qui elle-même suit la logique des structures sociales de la communauté. Les individus chez qui l'on retrouve plus de richesse, de façon générale, accèdent plus facilement aux postes publics des niveaux médians et majeurs. Les différents types de trajectoires qui amènent les paroissiens à s'engager dans les affaires publiques locales répondent donc à un ensemble de mécanismes de différenciation sociale (pistes 2 et 3). Certains clans familiaux se hissent au-dessus de la mêlée (piste 4). L'interaction entre les différents rôles publics locaux lève le voile sur une organisation publique à tout le moins complexe. Se révèle ainsi la dernière piste ouverte en début de ces pages, à savoir l'existence d'une structure étatique locale informelle (piste 5). Informelle parce qu'en partie considérée comme désorganisée, lâche et ténue par l'historiographie traditionnelle. Aussi, de considérer les structures de pouvoir d'avant 1840 comme un ensemble cohérent, fluide et articulé — avec ses propres codes et pratiques - relève d'un nouveau langage. Allan Greer avait d'ailleurs déjà reconnu cette complexité dans son travail sur les campagnes bas-canadiennes d'avant 1840 : «[...] la communauté rurale du Bas-Canada était bien autre chose $[\ldots]$ qu’un groupe atomisé de 
familles d'agriculteurs indépendantes, retenues ensemble par des institutions imposées [...] [elle] était en réalité d'une cohésion insoupçonnée ${ }^{81}$.»

Cette cohésion se réflète d'ailleurs dans la figure 2, qui expose les mécanismes inhérents au fonctionnement de cet appareil de pouvoir local. La mise à jour de ces mécanismes ouvre de nouvelles pistes de recherche, en permettant notamment d'approcher les rapports de force locaux avec de nouveaux outils de réflexion. Les réseaux de parentèle et de clientèle qui traversent les institutions aident à dévoiler les intérêts et les besoins des acteurs sociaux en place. Plus généralement, l'arrimage de la structure sociale à la structure institutionnelle permet de mieux comprendre l'évolution des formes institutionnelles, mais aussi le contraire, c'est-à-dire l'évolution des structures sociales dans les milieux ruraux.

Fort de la définition sociale de notre structure institutionnelle, il devient possible d'appréhender dans une perspective renouvelée la trame des conflits locaux qui jalonnent la période qui s'étend de 1810 à 1840. L'examen de l'appareil institutionnel au travers des secousses qu'il subit, autant de l'intérieur que de l'extérieur, risque d'apporter à son tour un éclairage sur les intérêts des acteurs sociaux en scène. L'intérêt réside justement dans le fait que la paroisse de Saint-Roch-de-l'Achigan ait été le théâtre de multiples déchirements, particulièrement autour du contrôle des revenus de la fabrique. L'analyse de cette trame nous permet de pousser plus avant la compréhension des mécanismes de production et reproduction de la condition élitaire locale, en même temps qu'elle offre un tremplin pour un chantier de réflexion dans une perspective régionale. Nous soupçonnons que les éléments les plus dynamiques des villages sont en réseau avec leurs semblables des autres bourgs de la région, de la même façon que certaines familles des districts de la paroisse Saint-Roch émergent pour alimenter une élite d'envergure locale. C'est à ce second volet que nous vous convions déjà. 